Krull-Gabriel dimension of 1-domestic string algebras

Prest, Mike and Puninski, Gena

2002

MIMS EPrint: 2006.124

Manchester Institute for Mathematical Sciences

School of Mathematics

The University of Manchester

\footnotetext{
Reports available from: http://eprints.maths.manchester.ac.uk/

And by contacting: The MIMS Secretary

School of Mathematics

The University of Manchester

Manchester, M13 9PL, UK
} 


\title{
KRULL-GABRIEL DIMENSION OF 1-DOMESTIC STRING ALGEBRAS
}

\author{
MIKE PREST AND GENA PUNINSKI
}

\begin{abstract}
We classify indecomposable pure injective modules over a wide class of 1-domestic string algebras and calculate the Krull-Gabriel dimension of these algebras.
\end{abstract}

\section{INTRODUCTION}

Finite dimensional string algebras over a field $\mathbb{k}$ form a particular class of path algebras of quivers with relations, and they are known for their rich representation theory. For instance every string algebra $A$ is tame, i.e. finite dimensional indecomposable representations of $A$ can be classified (see [2]). This result has interesting applications: the reader may consult $[19$, p. 653$]$ to find many examples.

As over any finite dimensional algebra, the indecomposable finite dimensional modules over a string algebra $A$ are organized into the Auslander-Reiten quiver, i.e. into a locally finite directed graph whose arrows are given by irreducible morphisms.

In [16] Ringel used some infinite dimensional indecomposable pure injective modules over a string algebra $A$ to glue together Auslander-Reiten components of $A$. If $A$ is a domestic string algebra, this leads to a nice geometric picture of the category of finite dimensional indecomposable $A$-modules.

In this context for a module $M$ to be pure injective means that $M$ is a direct summand of a direct product of finite dimensional $A$-modules. Obvious examples are the finite dimensional $A$-modules themselves: every such module is pure injective. Let us for simplicity assume that $\mathbb{k}$ is algebraically closed. Then for every band $C$ in the quiver of $A$ and every $0 \neq \lambda \in \mathbb{k}$ there is a $\lambda$-'Prüfer' module and a $\lambda$-'adic' module, which is pure injective and indecomposable. In addition there is a unique 'generic' indecomposable pure injective module corresponding to $C$. We will call these modules infinite dimensional band modules.

Less obvious examples were constructed by Ringel [16]. Precisely if $v$ is either a one-sided infinite string or a two-sided infinite (non-periodic) string over a domestic string algebra $A$ then there exists an indecomposable pure injective module $M(v)$ which is either the direct sum, direct product or a 'mixed' module.

2000 Mathematics Subject Classification. 16G20, 16D50.

Key words and phrases. Pure injective module, string algebra, Krull-Gabriel dimension.

This paper was written during the visit of the second author to the University of Manchester supported by EPSRC grant GR/R44942/01. He would like to thank the University for the kind hospitality. 
Conjecture 1.1. (Ringel - see $[16$, p. 48, p. 51]) Let $M$ be an infinite dimensional indecomposable pure injective module over a domestic string algebra. Then either $M$ is a band module, or $M$ is of the form $M(v)$ for a one-sided string or a two-sided (non-periodic) string $v$.

In [10] we proved that Ringel's conjecture is true for one-directed indecomposable pure injective modules over any domestic string algebra $A$. In this paper we verify Ringel's conjecture for 1-domestic string algebras of what we call type $\widetilde{A}$, i.e. for string algebras that have essentially one band and this band does not contain any repetition of vertices. Note that in general every domestic string algebra is $n$ domestic for some $n$.

To every domestic string algebra Schröer [17] has associated a finite directed graph without oriented cycles, the bridge quiver of $A$. It was conjectured by him that the Krull-Gabriel dimension of $A, \operatorname{KG}(A)$, is determined by its bridge quiver.

Conjecture 1.2. (Schröer - see [17, p. 84]) Let A be a domestic string algebra. Then the Krull-Gabriel dimension of $A$ is equal to $n+2$ where $n$ is the maximal length of a path in the bridge quiver of $A$.

It has been known since Geigle [4] that the Krull-Gabriel dimension of a hereditary tame finite dimensional algebra is equal to 2 . For instance this is the case for every (string) path algebra of an extended Dynkin diagram $\widetilde{A}_{k}$.

The inequality $\operatorname{KG}(A) \geq n+2$ for a domestic string algebra $A$ follows from [17]. Also (see [19] and [1]) for every $n \geq 2$ there is a domestic string algebra $A$ such that $\operatorname{KG}(A)=n$.

In this paper we prove that for a 1-domestic string algebra $A$ of type $\widetilde{A}$ either $n=0$ and then $\operatorname{KG}(A)=2$, or $n=1$ and then $\operatorname{KG}(A)=3$.

All these results are derived as consequences of a general approach originating in the model theory of modules. Rougly speaking this method allows one to classify indecomposable pure injective modules over a ring $R$, and to calculate their CantorBendixson rank in the Ziegler spectrum, provided we have a sufficient supply of distributive intervals in the lattice of finitely generated subfunctors of $\operatorname{Hom}(R,-)$.

Of course the existence of such a family of functors and the precise construction of them depends heavily on the description of indecomposable finitely presented $R$ modules and morphisms between them. In the case of string algebras this approach is aided by Crawley-Boevey's description [3] of morphisms between indecomposable string modules.

Instead of calculating the Krull-Gabriel dimension directly, we make the CantorBendixson analysis of the Ziegler spectrum over a 1-domestic string algebra $A$ to find its Cantor-Bendixson rank. After that a standard trick is used to show that it is equal to the Krull-Gabriel dimension of $A$. 


\section{String AlgEbras}

A module $M$ will usually mean a left module over a finite dimensional algebra $A$. Thus if $\alpha, \beta \in A$ and $m \in M$ then $(\alpha \beta) m=\alpha(\beta m)$. Also $M$ can be considered as a right module over its endomorphism $\operatorname{ring} S=\operatorname{End}(M)$.

String algebras form a particular class of path algebras of finite quivers with relations over a field $\mathbb{k}$. The precise definitions may be found in [2] or [18]. For instance

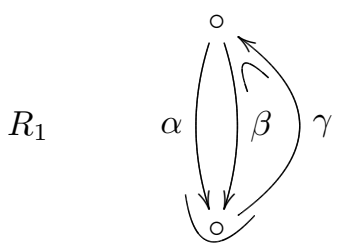

is a string algebra, where the zero relations, $\gamma \alpha=0$ and $\beta \gamma=0$, are indicated by short curves. In particular $R_{1}$ is an 8 -dimensional $\mathbb{k}$-vector space. Precisely, there are two primitive idempotents $e_{1}$ and $e_{2}$ corresponding to the vertices (i.e. two paths of length zero); three paths of length one: $\alpha, \beta, \gamma$; two paths of length two: $\alpha \gamma, \gamma \beta$; and one path of length three: $\alpha \gamma \beta$.

A string over a string algebra $A$ is a sequence $C=c_{1} \ldots c_{k}$ of arrows or inverse arrows reduced with respect to cancellation and such that neither a relation nor the inverse of a relation occurs in $C$. For instance $\alpha \beta^{-1} \alpha \gamma$ is a string over $R_{1}$ which is interpreted as 'go along $\gamma$, then along $\alpha$, lift through $\beta$, and go along $\alpha$ again'.

A key property of string algebras is that they are tame, i.e. admit a classification of the indecomposable finite dimensional modules. These modules are separated into two classes: string and band modules.

For every string $C$ over a string algebra $A$ there exists a string module $M(C)$. For instance for the string $C=\alpha \beta^{-1} \alpha \gamma$ the corresponding string module $M(C)$ has the following diagram:

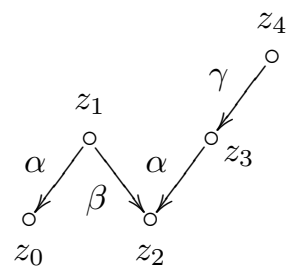

In particular $M(C)$ is a 5 -dimensional $\mathbb{k}$-vector space.

By [2] any string module $M(C)$ is indecomposable and $M(C) \cong M(D)$ iff either $C=D$ or $C=D^{-1}$.

A band over a string algebra $A$ is a string $C=c_{1} \ldots c_{k}$ such that the following holds:

1) every power $C^{m}$ is defined;

2) $C$ is not a power of a proper substring;

3) $c_{1}$ is a direct arrow and $c_{k}$ is an inverse arrow. 
Thus every band $C$ over $A$ is of the form $\alpha \ldots \beta^{-1}$. Note that then $C^{-1}=$ $\beta \ldots \alpha^{-1}$ is also a band and $C \neq C^{-1}$. For instance over $R_{1}$ the bands are $C=\alpha \beta^{-1}$ and $C^{-1}=\beta \alpha^{-1}$.

Let $C=c_{1} \ldots c_{k}$ be a band. For every $0 \neq \lambda \in \mathbb{k}$ and every $n>0$ we define a band module $M=M(C, \lambda, n)$ as follows. A $\mathbb{k}$-basis for $M$ is given by elements $z_{1}^{i}, \ldots, z_{k}^{i}, 1 \leq i \leq n$ and all actions are defined similarly to those for string modules $\left(c_{j}\right.$ acts between $z_{j}^{i}$ and $\left.z_{j+1}^{i}\right)$ with the unique exception of $j=k$. Here $\left(c_{k}=\beta^{-1}\right.$ acts between $z_{k}^{i}$ and $z_{1}^{i}$ ) we set $\beta z_{k}^{1}=\lambda z_{1}^{1}$ and $\beta z_{k}^{i}=\lambda z_{1}^{i}+z_{1}^{i-1}, 1<i \leq n$.

For instance for the band $C=\alpha \beta^{-1}$ over $R_{1}$ the corresponding band module $M(C, \lambda, 2)$ can be represented in the following way:

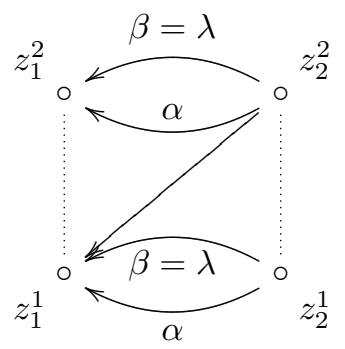

In view of [2] band modules $M(C, \lambda, m)$ and $M(D, \mu, n)$ are isomorphic iff $\lambda=\mu$, $m=n$, and $C$ is obtained from $D$ by a cyclic permutation and possibly taking the inverse.

We say that a string algebra $A$ is 1-domestic if there is a unique (up to cyclic permutation and inversion) band over $A$. For instance $R_{1}$ is 1 -domestic.

Let $R_{2}$ be the following string algebra [15, p. 49]:

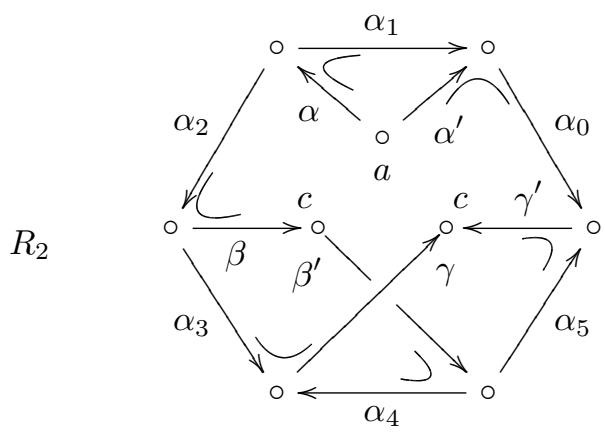

There is essentially one band over $R_{2}: C=\alpha_{4} \ldots \alpha_{3}^{-1}=\alpha_{0} \ldots \alpha_{5}^{-1}$ (we indentify bands that are obtained by a cyclic permutation), therefore $C^{-1}=\alpha_{3} \ldots \alpha_{4}^{-1}=$ $\alpha_{5} \ldots \alpha_{0}^{-1}$. Thus $R_{2}$ is 1 -domestic.

Usually for a 1-domestic string algebra $A$ we will fix a representative of the unique (up to cyclic permutation and inversion) band $C$. Then $C$ and $C^{-1}$ represent the bands over $A$ up to cyclic permutation. 


\section{BRIDGE QUIVER}

A one-sided word $v$ over a string algebra $A$ is a word of the form $c_{0} c_{1} \ldots$ or of the form $\ldots c_{-1} c_{0}$ where each $c_{i}$ is an arrow or an inverse arrow. We say that a one-sided word $v$ is a one-sided string if every finite subword of $v$ is a string. Similarly we can define a two-sided string $v=\ldots c_{-1} c_{0} c_{1} \ldots$

A one-sided string $v$ is said to be almost periodic if $v=C D^{\infty}$ for finite strings $C$ and $D$, or $v={ }^{\infty} C^{\prime} D^{\prime}$ for finite strings $C^{\prime}, D^{\prime}$. Similarly we say that a twosided string $v$ is biperiodic if $v={ }^{\infty} C D E^{\infty}$ for finite strings $C, D$ and $E$. Saying 'biperiodic' we usually exclude the case when $v$ is periodic, i.e. when $v$ can be represented in the form ${ }^{\infty} C^{\infty}$ for a finite string $C$.

Fact 3.1. [15, Prop. 2] Let A be a domestic string algebra. Then every one-sided string over $A$ is almost periodic, and every two-sided string over $A$ is biperiodic or periodic.

Note that if $C=\alpha \ldots \beta^{-1}$ is a band over any string algebra then $\alpha$ and $\beta$ are arrows ending in the same vertex. Since $\alpha \neq \beta$ (otherwise $C^{2}$ is not defined), $\alpha$ and $\beta$ determine each other uniquely. Let $B(\alpha)$ denote the set of all bands over $A$ with first letter $\alpha$.

Fact 3.2. $\left[19\right.$, L. 4.1] Let $C=\alpha \ldots \beta^{-1}$ be a band over a domestic string algebra $A$. Then $B(\alpha)$ consists of the one element $C$.

Lemma 3.3. Let $C=\alpha \ldots \beta^{-1}$, be a band over a domestic string algebra $A$. Then $C$ does not contain a proper substring which is a band.

Proof. Otherwise $C=E D F$ for a proper band $D=\mu \ldots \delta^{-1}$. By Fact 3.2 we may assume that $\mu \neq \alpha$ and $\delta \neq \beta$. Also the length of $D,|D|$, is less than the length of C.

We have $C^{2}=E D F E D F$. Since the string DFED begins with $\mu$ and ends with $\delta^{-1}$, by Fact 3.2 we obtain that $D F E D=D^{k}$ for some $k$. Then clearly $D=U \beta^{-1} \alpha V$ for some strings $U, V$. Thus $D^{2}=U \beta^{-1} \alpha V U \beta^{-1} \alpha V$, therefore (by Fact 3.2 again) $\alpha V U \beta^{-1}=C^{l}$ for some $l$. Then $|C| \leq\left|\alpha V U \beta^{-1}\right|=|D|$, a contradiction.

In general the structure of a band $C$ over even a 1-domestic string algebra may be quite complicated. Nevertheless there are some restrictions.

Lemma 3.4. Let $C=\alpha \ldots \beta^{-1}$ be a band over a domestic string algebra $A$. Then no arrow $\gamma$ occurs in $C$ twice as a direct arrow, and no arrow $\gamma$ occurs in $C$ twice as an inverse arrow.

Proof. Otherwise by symmetry we may assume that $\gamma$ occurs in $C$ twice as a direct arrow. If $\gamma=\alpha$, i.e. $C=\alpha E \alpha B \beta^{-1}$, then $\alpha B \beta^{-1}$ is a band which is shorter than $C$, a contradiction. 
Thus $C=E \gamma B \gamma D$ for some arrow $\gamma \neq \alpha$, where $E$ begins with $\alpha$ and $D$ ends with $\beta^{-1}$. Consider the word $C^{\prime}=E \gamma D$. Since $C^{\prime}$ begins with $\alpha$ and ends with $\beta^{-1}$ it cannot be a string (otherwise $C^{\prime}$ is a band shorter than $C$ ). Thus $C^{\prime}$ should contain a zero relation $E^{\prime} \gamma D^{\prime}$, i.e. $E=\ldots E^{\prime}, D=D^{\prime} \ldots$ and $E^{\prime}, D^{\prime}$ consist of direct arrows.

Since $C$ is a string, it does not contain $E^{\prime} \gamma D^{\prime}$ as a subword, therefore $E \gamma B \gamma \neq$ $\ldots E^{\prime} \gamma$, and $E \gamma=\ldots E^{\prime} \gamma$. Since $E^{\prime} \gamma$ consists of direct arrows, $E \gamma=\ldots \lambda \boldsymbol{\mu} E^{\prime \prime}$ and $E \gamma B \gamma=\ldots \delta^{-1} \mu E^{\prime \prime}$ for some (maybe empty) string $E^{\prime \prime}$. Thus $\delta$ and $\mu$ are arrows ending in the same vertex, and in $E \gamma B \gamma$ we obtain a configuration $\ldots \boldsymbol{\mu} \ldots \boldsymbol{\delta}^{-1} \ldots$ Thus $C$ properly contains a band $\mu \ldots \delta^{-1}$.

It remains to apply Lemma 3.3.

Note that the following string algebra

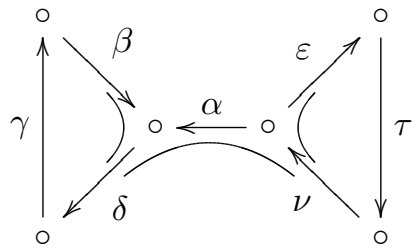

has a unique band $C=\alpha \nu \tau \varepsilon \alpha^{-1} \delta^{-1} \gamma^{-1} \beta^{-1}$ which contains a configuration $\alpha \ldots \alpha^{-1} \ldots$

Lemma 3.5. (see $\left[19\right.$, L. 4.1]) Let $C=\alpha \ldots \beta^{-1}$ be a band over a domestic string algebra $A$. Then $\alpha^{-1} \beta$ is not a substring of $C$.

Proof. Otherwise $C$ can be written as $\alpha B \alpha^{-1} \beta D \beta^{-1}$ for finite strings $B, D$, in particular $B$ is not empty. But then $C^{\prime}=\alpha B^{-1} \alpha^{-1} \beta D \beta^{-1}$ is a string. Since $B \neq B^{-1}$, therefore $C^{\prime} \neq C^{-1}$ is a band, a contradiction to Fact 3.2.

Lemma 3.6. Let $C=\alpha \ldots \beta^{-1}$ be a band over a domestic string algebra $A$. Then any distinct occurrences of $C$ in any string over $A$ have empty intersection. Also any occurrences of $C$ and $C^{-1}$ in any string over $A$ do not intersect each other.

Proof. $C$ cannot overlap $C$ in a non-trivial way by Lemma 3.4.

Suppose that $C$ overlaps $C^{-1}$ in a string $v$ over $A$. Since $C^{-1}=\beta \ldots \alpha^{-1}$, the configuration $C C^{-1}$ and $C^{-1} C$ in $v$ is not possible. By symmetry it suffices to consider the following configuration:

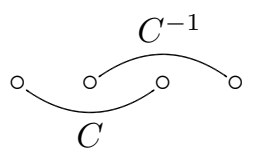

i.e. $v$ contains $\alpha E \beta F \beta^{-1} G \alpha^{-1}$, where $C=\alpha E \beta F \beta^{-1}$ and $C^{-1}=\beta F \beta^{-1} G \alpha^{-1}$. But then clearly $F=F^{-1}$, which gives a contradiction.

Corollary 3.7. Let $C=\alpha \ldots \beta^{-1}$ be a band over a domestic string algebra $A$ such that any relation on $A$ has length 2. Then the total number of occurrences of any arrow $\gamma$ (direct plus inverse) in $C$ is at most one. 
Proof. Otherwise by Lemma 3.4 and symmetry we may assume that $C=B \gamma D \gamma^{-1} E$, in particular $D$ is nonempty. Let $C^{\prime}=B \gamma D^{-1} \gamma^{-1} E$. Since all relations have length $2, C^{\prime}$ is a string. But clearly $D \neq D^{-1}$, therefore $C \neq C^{\prime}$, a contradiction to Fact 3.2 .

Over an arbitrary string algebra the classification of two-sided strings is hardly available. But for 1-domestic case we have the following description.

Lemma 3.8. (see $[16$, p. 58]) Let $A$ be a 1-domestic string algebra with a band $C=\alpha \ldots \beta^{-1}$. Then

1) every two-sided non-periodic string $v$ over $A$ can be written in the form $v=$ ${ }^{\infty} B^{-1} D B^{\infty}$, where $B=C$ or $C^{-1}$ and the length of $D$ is uniformly bounded. Moreover this representation of $v$ is unique, if $D$ is of minimal length.

2) Every one-sided string $w$ over $A$ can be written in the form $w=E B^{-n} F B^{\infty}$ or $w={ }^{\infty} B^{-1} G B^{m} H$, where $B=C$ or $C^{-1}$ and the lengths of $E, F, G, H$ are uniformly bounded. This representation is also unique, if $E, F, G, H$ are of minimal lengths.

Proof. Let $v$ be a two-sided string over $A$.

First there exists an $m$ such that every string of length $m$ over $A$ contains a sink, i.e. a substring of the form $\gamma^{-1} \delta$. Let us cut $v$ into pieces of length $m$. Also there exist only finitely many, say $n$, possibilities for sinks. Since $A$ is 1-domestic, by Fact 3.2 a configuration $\gamma^{-1} \delta \ldots \gamma^{-1} \delta$ in $v$ implies that the dotted part must be filled in by a power of a (unique) band $D=\delta \ldots \gamma^{-1}$ (and $D$ is a cyclic permutation of either $C$ or $C^{-1}$ ).

Since at least one sink will appear eventually on the left, the left hand part of $v$ is of the form ${ }^{\infty} E$, and the right hand part of $v$ is of the form $F^{\infty}$, where $E, F$ are bands. If $E=F$ (up to cyclic permutation) then (by Fact 3.2) $v$ is periodic. Otherwise we may assume that $E=C^{-1}, F=C$ and $v={ }^{\infty} C^{-1} G C^{\infty}$, where the length of $G$ is minimal possible.

We prove that the length of $G$ bounded above by $m(2 n+1)$. Indeed otherwise there are three appearances in $G$ of the same sink, therefore a square of a band $H^{2}$ occurs in $G$. Since $H$ is a cyclic permutation of either $C$ or $C^{-1}$, one of these bands occurs in $G$. By Fact 3.2 this contradicts the minimality of $G$.

Now the uniqueness follows by Lemma 3.6.

If $w$ is a one-sided string, the proof is similar.

Corollary 3.9. Let $A$ be a 1-domestic string algebra. Then there exist only finitely many two-sided strings over $A$.

Proof. This follows from Lemma 3.8.

Let $A$ be a domestic string algebra. In [19] Schröer defined the bridge quiver of $A$ to be a finite oriented graph on the set of bands of $A$. If $A$ is 1-domestic, this graph can be introduced very easily. Let $C$ and $C^{-1}$ be representatives (up to 
cyclic permutation) of the bands over $A$. Let $v$ be a two-sided string over $A$ written as $v={ }^{\infty} B^{-1} D B^{\infty}$, where $B=C$ or $B=C^{-1}$, and $D$ contains neither $C$ nor $C^{-1}$ as a substring. Then we include in the bridge quiver the arrow $B^{-1} \rightarrow B$ with the label $D$.

For instance

$$
w={ }^{\infty}\left(\beta^{-1} \alpha\right) \gamma\left(\beta \alpha^{-1}\right)^{\infty}
$$

is a two-sided string over $R_{1}$ which is essentially unique: every (finite or infinite) string over $R_{1}$ is a substring of either $w$ or $w^{-1}$. From $w$ we obtain an arrow

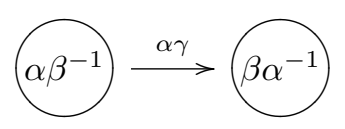

and inverting this

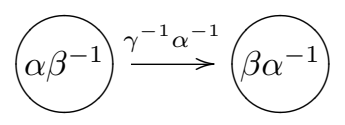

Thus the bridge quiver of $R_{1}$ is $C \stackrel{2}{\longrightarrow} C^{-1} \quad$ (two arrows between $C$ and $C^{-1}$ ).

Also up to inversion there are only three two-sided strings over $R_{2}$ :

$$
\begin{gathered}
z(a)={ }^{\infty}\left(\alpha_{1}^{-1} \alpha_{0}^{-1} \alpha_{5} \alpha_{4}^{-1} \alpha_{3} \alpha_{2}\right) \alpha \alpha^{\prime-1}\left(\alpha_{1} \alpha_{2}^{-1} \alpha_{3}^{-1} \alpha_{4} \alpha_{5}^{-1} \alpha_{0}\right)^{\infty}, \\
z(b)={ }^{\infty}\left(\alpha_{2} \alpha_{1}^{-1} \alpha_{0}^{-1} \alpha_{5} \alpha_{4}^{-1} \alpha_{3}\right) \beta^{-1} \beta^{-1}\left(\alpha_{5}^{-1} \alpha_{0} \alpha_{1} \alpha_{2}^{-1} \alpha_{3}^{-1} \alpha_{4}\right)^{\infty},
\end{gathered}
$$

and

$$
z(c)={ }^{\infty}\left(\alpha_{3} \alpha_{2} \alpha_{1}^{-1} \alpha_{0}^{-1} \alpha_{5} \alpha_{4}^{-1}\right) \gamma^{-1} \gamma^{\prime}\left(\alpha_{0} \alpha_{1} \alpha_{2}^{-1} \alpha_{3}^{-1} \alpha_{4} \alpha_{5}^{-1}\right)^{\infty}
$$

From $z(a)$ we obtain the arrow

$$
\alpha_{3} \ldots \alpha_{4}^{-1} \stackrel{\alpha_{3} \alpha_{2} \alpha \alpha^{\prime-1} \alpha_{1} \alpha_{2}^{-1} \alpha_{3}^{-1}}{\longrightarrow} \alpha_{4} \ldots \alpha_{3}^{-1}
$$

and $z(b)$ leads to the arrow

$$
\alpha_{3} \ldots \alpha_{4}^{-1} \stackrel{\alpha_{3} \beta^{-1} \beta^{\prime-1} \alpha_{5}^{-1}}{\longrightarrow} \alpha_{0} \ldots \alpha_{5}^{-1}
$$

Finally $z(c)$ gives the arrow

$$
\alpha_{3} \ldots \alpha_{4}^{-1} \stackrel{\gamma^{-1} \gamma^{\prime}}{\longrightarrow} \alpha_{0} \ldots \alpha_{5}^{-1}
$$

Inverting these we obtain that the bridge quiver of $R_{2}$ is $C^{-1} \stackrel{6}{\longrightarrow} C$.

Lemma 3.10. Let $A$ be a 1-domestic string algebra with the band $C$. Then the bridge quiver of $A$ is either $B^{-1} \rightarrow B$, or a disjoint union of $C$ and $C^{-1}$, where $B=C$ or $B=C^{-1}$. 
Proof. By symmetry it suffices to prove that the case $C^{-1} \rightarrow C \rightarrow C^{-1}$ is not possible. Otherwise there are two-sided strings ${ }^{\infty}\left(C^{-1}\right) E C^{\infty}$ and ${ }^{\infty} C F\left(C^{-1}\right)^{\infty}$. But then $v={ }^{\infty}\left(C^{-1}\right) E C F\left(C^{-1}\right)^{\infty}$ is also a string. By Lemma 3.8 we may write $v$ as ${ }^{\infty}\left(B^{-1}\right) G B^{\infty}$, where $B=C$ or $B=C^{-1}$. But by Lemma $3.6 C$ and $C^{-1}$ cannot overlap each other, a contradiction.

For instance if $A$ is a hereditary tame finite dimensional algebra of type $\widetilde{A}_{n}$, then the bridge quiver of $A$ is the disjoint union of $C$ and $C^{-1}$. Note also that the bridge quiver of a 1-domestic string algebra $A$ is nontrivial (i.e. contains an arrow) iff there is a two-sided nonperiodic string over $A$.

\section{Distributive intervals}

Basic notions from the model theory of modules can be found in [6]. Also see [10] for a summary.

Saying that $(\varphi / \psi)$ is a pair of pp-formulae we usually assume that $\psi<\varphi$. The notation $(\varphi / \psi)$ will be also used for the interval $[\psi ; \varphi]$ in the lattice of all ppformulae over $A$. A pair $(\varphi / \psi)$ opens a module $M$ if $\varphi(M) / \psi(M)$ is nonzero. These subgroups and their factors are right $S$-submodules of $M$ where $S=\operatorname{End}(M)$.

The Ziegler spectrum over $A, \mathrm{Zg}_{A}$, is a topological space whose points are (isomorphism types of) indecomposable pure injective $A$-modules. The topology on $\mathrm{Zg}_{A}$ is given by basic opens sets $(\varphi / \psi)=\left\{M \in \mathrm{Zg}_{A} \mid(\varphi / \psi)\right.$ opens $\left.M\right\}$, and this is the third meaning of $(\varphi / \psi)$. This space is (quasi) compact, and by [6, Prop. 13.1] the isolated points of $\mathrm{Zg}_{A}$ are exactly the indecomposable finite dimensional $A$ modules.

For every pp-type $p$ there exists a 'minimal' pure injective module $N(p)$ and an element $m \in N(p)$ such that the pp-type of $m$ in $N(p), p p_{N(p)}(m)$, is equal to $p$. A pp-type $p$ is indecomposable if the module $N(p)$ is indecomposable.

For an indecomposable pp-type $p$ we write $p \in(\varphi / \psi)$ if $\varphi \in p$ and $\psi \in p^{-}$. Then $p$ defines a cut on the interval $(\varphi / \psi)$ in the obvious way: we take $\theta \in(\varphi / \psi)$ in the 'upper part' of the cut if $\theta \in p^{+}$and take $\theta$ in the 'lower part' of the cut if $\theta \in p^{-}$:

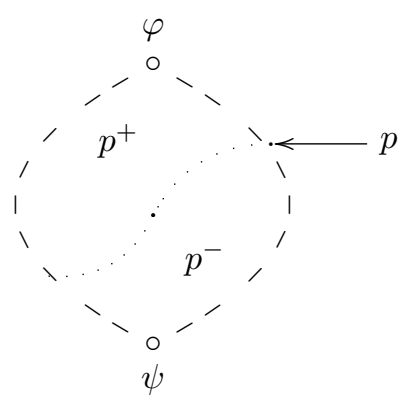

Fact 4.1. (see $[6$, L. 9.2]) Let $(\varphi / \psi)$ be a pair of pp-formula and let $p, q \in(\varphi / \psi)$ be indecomposable pp-types which define the same cut on $(\varphi / \psi)$. Then $N(p) \cong N(q)$.

Thus, the module $N(p)$ is uniquely determined by any nontrivial cut of $p$. The global shape of an indecomposable pp-type is given by Ziegler's criterion. 
Fact 4.2. [6, Thm. 4.29] A pp-type $p$ is indecomposable iff for every $\psi_{1}, \psi_{2} \in p^{-}$ there exist $\varphi \in p^{+}$such that $\left(\psi_{1} \wedge \varphi\right)+\left(\psi_{2} \wedge \varphi\right) \in p^{-}$.

Nevertheless it is not clear how to describe (in lattice theoretical terms) cuts on an interval given by indecomposable pp-types.

We say that a pair $(\varphi / \psi)$ is distributive if the interval $(\varphi / \psi)$ is a distributive lattice (i.e. the lattice of all pp-formulae between $\varphi$ and $\psi$ is distributive).

Our next aim is to prove that an indecomposable pp-type defines a filter-cofilter partition on every distributive interval. It is not clear how to prove it directly, since some 'external' formulae may intrude when we try to apply Ziegler's criterion.

So we should make a digression first.

A module $M$ is distributive if the lattice of submodules of $M$ is distributive. For instance every uniserial module is distributive. On the other hand $M \oplus M$ is never distributive for a nonzero $M$ (consider the diagonal embedding). Recall first the well known characterization of distributivity [20, Prop. 1.17]. A right module $M$ over a ring $S$ is distributive iff for every $m, n \in M$ there are $f, g, h \in S$ such that $m f=n g$ and $n(1-f)=m h$.

Lemma 4.3. Let $(\varphi / \psi)$ be a pair of pp-formulae. For every module $M$ we consider the following property $(*): \varphi(M) / \psi(M)$ is a distributive $S$-module where $S=\operatorname{End}(M)$. Then $(*)$ is preserved under forming arbitrary direct sums and products of modules, and under taking direct summands.

Proof. This is a slight modification of $[20,8.6]$. Let $M_{i}, i \in I, S_{i}=\operatorname{End}\left(M_{i}\right)$ be such that $(*)$ holds for every $M_{i}$ and let $M=\prod_{i \in I} M_{i}, S=\operatorname{End}(M)$. Take any $m=\left(m_{i}\right), n=\left(n_{i}\right) \in \varphi(M)$, therefore $m_{i}, n_{i} \in \varphi\left(M_{i}\right)$ for every $i$. By assumption, for every $i$ there are $f_{i}, g_{i}, h_{i} \in S_{i}$ such that $m_{i} f_{i}=n_{i} g_{i}+k_{i}$ and $n_{i}\left(1-f_{i}\right)=$ $m_{i} h_{i}+l_{i}, k_{i}, l_{i} \in \psi\left(M_{i}\right)$. Then $f=\left(f_{i}\right), g=\left(g_{i}\right), h=\left(h_{i}\right)$ are endomorphisms of $M$, and $m f=n g+k, n(1-f)=m h+l$, where $k=\left(k_{i}\right), l=\left(l_{i}\right)$ are clearly in $\psi(M)$.

The proof when $M$ is the direct sum of the $M_{i}$ is the same.

Suppose that $M$ is a direct summand of a module $N$ with $(*), S=\operatorname{End}(N)$, and let $e$ be the canonical projection onto $M$, in particular $\operatorname{End}(M)=e S e$. Take any $m, n \in \varphi(M)$, therefore $m e=m$ and $n e=n$. By assumption, since $m, n \in \varphi(N)$, there are $f, g, h \in S$ such that $m f=n g+k$ and $n(1-f)=m h+l, k, l \in \psi(N)$. Multiplying this by $e$ on the right we obtain mefe $=n e g e+k e, n e(e-e f e)=$ mehe + le where efe, ege, ehe are endomorphisms of $M$ and $k e, l e \in \psi(M)$.

Proposition 4.4. Let $(\varphi / \psi)$ be a pair of pp-formulae over A. Then the following assertions are equivalent:

1) $(\varphi / \psi)$ is distributive;

2) $\varphi(M) / \psi(M)$ is a distributive $S$-module for every pure injective module $M$ where $S=\operatorname{End}(M)$; 
3) $\varphi(M) / \psi(M)$ is a uniserial $S$-module for every indecomposable pure injective module $M$ where $S=\operatorname{End}(M)$;

4) $\varphi(M) / \psi(M)$ is a distributive $S$-module for every finite dimensional module $M$ where $S=\operatorname{End}(M)$;

5) $\varphi(M) / \psi(M)$ is a uniserial $S$-module for every indecomposable finite dimensional module $M$ where $S=\operatorname{End}(M)$.

Proof. 1) $\Rightarrow 4$ ). By [20, Prop. 1.17] it suffices to check distributivity for cyclic $S$-submodules of $\varphi(M) / \psi(M)$. Thus it is enough to check distributivity for $S$ submodules of $M$ of the form $m S+\psi(M), m \in \varphi(M)$. By [6, Prop. 8.4] $m S=$ $\theta(M)$ for some $\theta$ (generating the pp-type of $m$ in $M$, in particular $\theta \rightarrow \varphi$ ). Thus $m S+\psi(M)=(\theta+\psi)(M)$ and this formula is clearly between $\psi$ and $\varphi$.

$4) \Rightarrow 5)$. By [20, Thm. 1.21] every distributive module over a local ring is uniserial. By similar arguments 2) implies 3).

5) $\Rightarrow 4$ ) by Lemma 4.3 .

Similarly 4$) \Rightarrow 2$ ) follows, since every pure injective module is a direct summand of a direct product of indecomposable finite dimensional modules (because $A$ is finite dimensional).

$2) \Rightarrow 1$ ). The lattice of all pp-formulae over $A$ is the lattice of pp-subgroups of a 'large' pure injective module $M$. Since $\varphi(M) / \psi(M)$ is a distributive $S$-module, the result follows.

Also it is clear that 3$) \Rightarrow 5$ ).

Note that this result is true, except for 5), over an arbitary ring $R$ if we replace 'finite dimensional module' in 4) by 'finitely presented module'. 5) can be included if every finitely presented module over $R$ is a direct sum of modules with local endomorphism rings.

Now we return to our earlier considerations.

Lemma 4.5. Let $(\varphi / \psi)$ be a distributive pair of pp-formulae and let $p \in(\varphi / \psi)$ be an indecomposable pp-type. Then $p$ defines a filter-cofilter partition on the interval $(\varphi / \psi)$, and the module $N(p)$ is uniquely determined by this cut. Moreover for every filter-cofilter partition of $(\varphi / \psi)$ there exists an indecomposable pp-type which defines this cut.

Proof. Let $M=N(p)$ (an indecomposable pure injective module) and let $m \in M$ realize $p$. It suffices to prove that the lower part of the cut, $p^{-}$, is closed with respect to sums. Let $\varphi_{1}, \varphi_{2} \in p^{-}$with $\varphi \leq \varphi_{1}, \varphi_{2} \leq \psi$, therefore $m \notin \varphi_{1}(M), \varphi_{2}(M)$. By Proposition 4.4 the $S$-modules $\varphi_{1}(M), \varphi_{2}(M)$ are comparable, therefore we may assume that $\varphi_{1}(M) \subseteq \varphi_{2}(M)$. Then from $m \notin\left(\varphi_{1}+\varphi_{2}\right)(M)=\varphi_{2}(M)$ it follows that $\varphi_{1}+\varphi_{2} \in p^{-}$.

$N(p)$ is uniquely determined by the cut, by Fact 4.1.

So it remains to prove that every filter-cofilter partition of the interval $(\varphi / \psi)$ is defined by some indecomposable pp-type. Let us put the upper part of this 
partition into $p^{+}$and the lower part of this partition into $p^{-}$. Clearly the set of formulae $p^{+} \cup \neg p^{-}$is consistent. Now we extend this pp-type to a maximal pptype $q$ including $p^{+}$and omitting $p^{-}$. From [6, Thm. 4.33] it follows that $q$ is indecomposable, and clearly $q$ defines the required cut.

Thus we may roughly classify indecomposable pure injective modules living on a distributive interval $(\varphi / \psi)$ by their cuts (caution: different cuts may lead to the same module).

Proposition 4.6. Let $(\varphi / \psi)$ be a distributive interval in the lattice of all ppformulae over $A$. Then there exists a natural surjection from the set of filter-cofilter partitions of this interval to the set of isomorphism types of indecomposable pure injective modules opening $(\varphi / \psi)$.

Proof. This follows from Lemma 4.5.

If the more precise structure of the interval $(\varphi / \psi)$ is known, a more satisfactory analysis is possible.

Let $L$ and $L^{\prime}$ be chains with largest and smallest elements. By $L \otimes L^{\prime}$ we will denote the modular lattice freely generated by $L$ and $L^{\prime}$ with additional relations $0=0^{\prime}$ and $1=1^{\prime}$ (i.e. the smallest elements of $L$ and $L^{\prime}$ are identified and the same is done with the largest elements). It is well known (see [5]) that this lattice is distributive (and a quite satisfactory description of its elements is possible - see [12] for an explanation).

We say that the interval $(\varphi / \psi)$ in the lattice of all pp-formulae over $A$ is generated by the chains $L_{1}$ and $L_{2}$ if 1) $L_{1}$ and $L_{2}$ consist of formulae between $\psi$ and $\varphi ; 2$ ) $0_{1}=0_{2}=\psi, 1_{1}=1_{2}=\varphi$, and 3) every formula between $\psi$ and $\varphi$ is in the lattice generated by $L_{1} \cup L_{2}$.

Similarly an interval $(\varphi / \psi)$ is freely generated by the chains $L_{1}$ and $L_{2}$ if in addition 4) the natural epimorphism $L_{1} \otimes L_{2} \rightarrow(\varphi / \psi)$ is an isomorphism.

Suppose that the interval $(\varphi / \psi)$ is generated by chains $L_{1}, L_{2}$ and let $p \in(\varphi / \psi)$ be an indecomposable pp-type. Define $p_{1}$ to be the cut on $L_{1}$ determined by $p$, i.e. $\varphi^{\prime} \in L_{1}$ is in the upper part of $p_{1}, p_{1}^{+}$, if $\varphi^{\prime} \in p$, and $\varphi^{\prime \prime} \in L_{1}$ is in the lower part of $p_{1}, p_{1}^{-}$, if $\varphi^{\prime \prime} \in p^{-}$. Similarly $p$ defines a cut $p_{2}$ on $L_{2}$.

Lemma 4.7. Let an interval $(\varphi / \psi)$ be generated by chains $L_{1}, L_{2}$ and let $p \in(\varphi / \psi)$ be an indecomposable pp-type. Then the cut of $p$ is uniquely determined by its cuts on $L_{1}$ and $L_{2}$, i.e. by $p_{1}$ and $p_{2}$.

Proof. By assumption every pp-formula $\theta$ between $\psi$ and $\varphi$ is equivalent to a formula $\sum_{i} \varphi_{i} \wedge \psi_{i}$ where $\varphi_{i} \in L_{1}$ and $\psi_{i} \in L_{2}$. By Lemma 4.5, $p$ defines a filter-cofilter partition on $(\varphi / \psi)$. Therefore $\theta \in p$ iff $\varphi_{i} \wedge \psi_{i} \in p$ for some $i$, i.e. if $\varphi_{i} \in p_{1}^{+}$and $\psi_{i} \in p_{2}^{+}$.

So an indecomposable pp-type $p$ living on a distributive interval generated by chains $L_{1}, L_{2}$ may be drawn as follows (a marked point means that $\varphi^{\prime} \wedge \psi^{\prime} \in p$ ) 


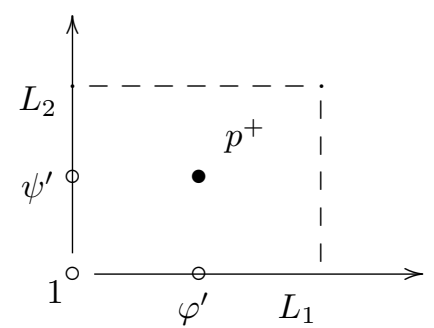

In view of Lemma 4.7 we will write $p=\left(p_{1}, p_{2}\right)$ for an indecomposable pp-type $p$, i.e. $p$ may be considered as a pair of cuts. Not every pair of cuts leads to an indecomposable pp-type. We say that a pair of cuts $\left(p_{1}, p_{2}\right)$ is admissible if there is an indecomposable pp-type $p$ that defines this pair.

Lemma 4.8. A pair of cuts $\left(p_{1}, p_{2}\right)$ is admissible iff $\varphi^{\prime} \wedge \psi^{\prime}$ does not imply $\varphi^{\prime \prime}+\psi^{\prime \prime}$ for every $\varphi^{\prime}, \varphi^{\prime \prime} \in L_{1}, \psi^{\prime}, \psi^{\prime \prime} \in L_{2}$ such that $\varphi^{\prime} \in p_{1}, \varphi^{\prime \prime} \in p_{1}^{-}, \psi^{\prime} \in p_{2}, \psi^{\prime \prime} \in p_{2}^{-}$.

Proof. Assume that there exists an indecomposable pp-type $p$ defining the pair $\left(p_{1}, p_{2}\right)$ such that $\varphi^{\prime} \wedge \psi^{\prime} \rightarrow \varphi^{\prime \prime}+\psi^{\prime \prime}$. Then $\varphi^{\prime} \in p_{1}, \psi^{\prime} \in p_{2}$ implies that $\varphi^{\prime} \wedge \psi^{\prime} \in p$, therefore $\varphi^{\prime \prime}+\psi^{\prime \prime} \in p$. By Lemma 4.5 it follows that either $\varphi^{\prime \prime} \in p$ or $\psi^{\prime \prime} \in p$. But $\varphi^{\prime \prime} \in p$ implies $\varphi^{\prime \prime} \in p_{1}$, a contradiction. Similarly $\psi^{\prime \prime} \in p$ yields $\psi^{\prime \prime} \in p_{2}$, a contradiction again.

For the converse put into $p^{+}$all pp-formulae $\varphi^{\prime} \wedge \psi^{\prime}$ where $\varphi^{\prime} \in p_{1}, \psi^{\prime} \in p_{2}$, and put into $p^{-}$all pp-formulae $\varphi^{\prime \prime}+\psi^{\prime \prime}$ where $\varphi^{\prime \prime} \in p_{1}^{-}$and $\psi^{\prime \prime} \in p_{2}^{-}$. Clearly $p^{+}$ is closed with respect to finite conjunctions and $p^{-}$is closed with respect to finite sums. Therefore by the assumption $p^{+} \cup \neg p^{-}$is a consistent set of pp-formulae. Extend this set to a maximal pp-type $q$ containing $p^{+}$and omitting $p^{-}$.

Then $q$ is indecomposable and clearly $q_{1}=p_{1}, q_{2}=p_{2}$.

Therefore we obtain the following:

Lemma 4.9. Let an interval $(\varphi / \psi)$ be generated by chains $L_{1}$ and $L_{2}$. Then there is one-to-one correspondence between cuts on $(\varphi / \psi)$ given by indecomposable pptypes and admissible pairs of cuts $\left(p_{1}, p_{2}\right)$ on $L_{1}$ and $L_{2}$. If $(\varphi / \psi)$ is freely generated by $L_{1}$ and $L_{2}$ then every pair of cuts is admissible.

Proof. By Lemma 4.8 every indecomposable pp-type $p \in(\varphi / \psi)$ defines an admissible pair of cuts $\left(p_{1}, p_{2}\right)$. Also by Lemma 4.7 the cut of $p$ is uniquely determined by this pair.

Let $(\varphi / \psi)$ be freely generated by $L_{1}, L_{2}$. Then by Lemma 4.8 and the description of elements of the lattice freely generated by $L_{1}$ and $L_{2}$, every pair of cuts $\left(p_{1}, p_{2}\right)$ is admissible.

Thus indecomposable pure injective modules living in an open set $(\varphi / \psi)$ generated by chains $L_{1}, L_{2}$ are classified by admissible pairs of cuts $\left(p_{1}, p_{2}\right)$ on $L_{1}$ and $L_{2}$. 
Proposition 4.10. Let an interval $(\varphi / \psi)$ be generated by chains $L_{1}$ and $L_{2}$. Then there exists a natural surjection from the set of admissible pairs of cuts $\left(p_{1}, p_{2}\right)$ on $L_{1}$ and $L_{2}$ to the set of isomorphism types of indecomposable pure injective modules opening $(\varphi / \psi)$.

Proof. This follows from Lemma 4.9 and Proposition 4.6.

\section{Cantor-Bendixson analysis}

Let $L$ be a modular lattice with smallest element 0 and largest element 1 . Recall that the $m$-dimension of $L, \operatorname{mdim}(L)$, is defined by iterated factorization by the equivalence relation which collapses intervals of finite length. For instance $\operatorname{mdim}(L)=0$ iff $L$ is finite and $\operatorname{mdim}(\omega+1)=1$. Also the $m$-dimension of $L$ is undefined iff $L$ contains the order type of the rationals $\mathbb{Q}$ as a subchain.

Sometimes it is possible to calculate the $m$-dimension of a lattice directly.

Let ordinals $\lambda$ and $\mu$ be given in their Cantor normal form: $\lambda=\omega^{\alpha_{1}} n_{1}+\cdots+$ $\omega^{\alpha_{k}} n_{k}, \mu=\omega^{\alpha_{1}} m_{1}+\cdots+\omega^{\alpha_{k}} m_{k}, \alpha_{1}>\cdots>\alpha_{k}$. Then $\lambda \oplus \mu$ will denote the ordinal $\omega^{\alpha_{1}}\left(n_{1}+m_{1}\right)+\cdots+\omega^{\alpha_{k}}\left(n_{k}+m_{k}\right)$. For instance $1 \oplus \omega=\omega \oplus 1=\omega+1$.

Fact 5.1. [13, Prop. 4.2] Let $L, L^{\prime}$ be chains such that $\operatorname{mdim}(L)=\lambda, \operatorname{mdim}\left(L^{\prime}\right)=$ $\lambda^{\prime}$. Then $\operatorname{mdim}\left(L \otimes L^{\prime}\right)=\lambda \oplus \lambda^{\prime}$.

Now we describe the Cantor-Bendixson, $\mathrm{CB}$, analysis on the interval generated by two chains. Let an interval $(\varphi / \psi)$ be generated by chains $L_{1}, L_{2}$, and let $p \in(\varphi / \psi)$ be an indecomposable pp-type. From Lemma 4.9 it follows that the cut of $p$, therefore the module $N(p)$, is completely determined by the corresponding pair $\left(p_{1}, p_{2}\right)$ of its cuts on the chains $L_{1}$ and $L_{2}$. As in [10] let us define $\operatorname{mdim}_{1}(p)$ as the infimum of $m$-dimensions of chains $\left(\varphi^{\prime} / \psi^{\prime}\right), \varphi^{\prime}, \psi^{\prime} \in L_{1}$ such that $p \in\left(\varphi^{\prime} / \psi^{\prime}\right)$. Similarly define $\operatorname{mdim}_{2}(p)$.

Also let the $m$-dimension of $p, \operatorname{mdim}(p)$, be the infimum of $m$-dimensions of intervals $\left(\varphi_{1} / \psi_{1}\right)$ such that $\psi \leq \psi_{1}<\varphi_{1} \leq \varphi$ and $p \in\left(\varphi_{1} / \psi_{1}\right)$.

Lemma 5.2. Let an interval $(\varphi / \psi)$ in the lattice of all pp-formulae over $A$ be generated by chains $L_{1}$ and $L_{2}$, and let $p \in(\varphi / \psi)$ be an indecomposable pp-type. Then $\mathrm{CB}(p)=\operatorname{mdim}(p) \leq \operatorname{mdim}_{1}(p) \oplus \operatorname{mdim}_{2}(p)$. Moreover if $(\varphi / \psi)$ is freely generated by $L_{1}$ and $L_{2}$ then $\mathrm{CB}(p)=\operatorname{mdim}_{1}(p) \oplus \operatorname{mdim}_{2}(p)$.

Proof. From [13, Thm. 3.1] it follows that the isolation property holds for $(\varphi / \psi)$ : for any theory $T$ of $A$-modules every isolated point in $T \cap(\varphi / \psi)$ is isolated by a minimal pair. Then similarly to [6, Prop. 10.19] we obtain that $\operatorname{mdim}(p)=\mathrm{CB}(p)$ for every indecomposable pp-type $p \in(\varphi / \psi)$.

First assume that $(\varphi / \psi)$ is freely generated by $L_{1}$ and $L_{2}$. Let $M=N(p)$ (thus $M$ is an indecomposable pure injective module) and let $m$ realize $p$ in $M$.

Since $(\varphi / \psi)$ is an open set in the Ziegler spectrum of $A$, the Cantor-Bendixson analysis can be carried out inside $(\varphi / \psi)$. 
Clearly a basis of open sets (in $\mathrm{Zg}_{A}$ ) for $M$ can be chosen to be of the following form: $\left(\varphi^{\prime} \wedge \psi^{\prime} / \varphi^{\prime \prime}+\psi^{\prime \prime}\right)$, where $\varphi^{\prime}, \varphi^{\prime \prime} \in L_{1}, \psi^{\prime}, \psi^{\prime \prime} \in L_{2}$ and $\varphi^{\prime}, \psi^{\prime} \in p, \varphi^{\prime \prime}, \psi^{\prime \prime} \in p^{-}$.

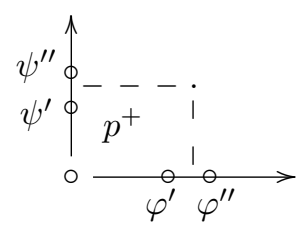

By a fairly standard procedure (see [14] for a similar analysis over a serial ring) one shows that the CB-rank of $M$ is equal to $\operatorname{mdim}_{1}(p) \oplus \operatorname{mdim}_{2}(p)$. For instance $M$ is isolated iff $p$ defines principal cuts (i.e. if the positive part of $p_{1}$ is generated by one pp-formula and the negative part of $p_{1}$ is generated by one pp-formula; and similarly for $\left.p_{2}\right)$. Also $M$ is of CB-rank 1 if either $p_{1}$ is principal and $p_{2}$ has $m$-dimension $1(0+1=1)$ or $p_{1}$ has $m$-dimension 1 and $p_{2}$ is principal $(1+0=1)$.

If $(\varphi / \psi)$ is not freely generated by $L_{1}$ and $L_{2}$, the same analysis gives the upper bound for the CB-rank of every pp-type $p \in(\varphi / \psi): \operatorname{CB}(p) \leq \operatorname{mdim}\left(p_{1}\right) \oplus \operatorname{mdim}\left(p_{2}\right)$.

\section{IndECOMPOSABLE PURE INJECTIVE MODULES}

Recall that every indecomposable finite dimensional $A$-module is pure injective, therefore it is a point in $\mathrm{Zg}_{A}$.

Let $A$ be a 1 -domestic string algebra with a (unique) band $C=\alpha \ldots \beta^{-1}$. We say that $A$ is of type $\widetilde{A}$, if no vertex (except at the ends) occurs twice in $C$. Therefore the subquiver corresponding to $C$ is the quiver of a tame hereditary finite dimensional algebra of type $\widetilde{A}$.

Note that in many examples (see Corollary 3.7) $C$ does not contain a repetition of an arrow. But even in this case the structure of $C$ may be complicated. A typical algebra with a repetition of a vertex is

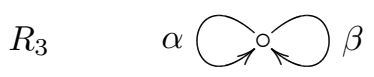

with the relation $\alpha^{2}=\beta^{2}=\beta \alpha=0$. Clearly $C=\alpha \beta^{-1}$ is a unique band over $A$. Also every string over $R_{3}$ is (up to inversion) a substring of

$$
{ }^{\infty}\left(\beta^{-1} \alpha\right)\left(\beta \alpha^{-1}\right)^{\infty}
$$

therefore $R_{3}$ is 1 -domestic. $R_{3}$ is not of type $\widetilde{A}$, since the band $\alpha \beta^{-1}$ has just one vertex.

Let $A$ be a 1-domestic string algebra of type $\widetilde{A}$ with band $C$. Let $B$ be the corresponding tame hereditary algebra of type $\widetilde{A}$. Then there is a natural representation embedding $f$ from the category of $B$-modules into the category of $A$-modules 'living' on $C$.

Lemma 6.1. Let $A$ be a 1-domestic string algebra of type $\widetilde{A}$ with band $C=$ $\alpha \ldots \beta^{-1}$. Then $f$ induces a homeomorphism from $\mathrm{Zg}_{B}$ onto a closed subset $\mathcal{A}$ 
of $\mathrm{Zg}_{A}$. Also $\mathcal{A}$ consists of the indecomposable pure injective modules $M$ such that $e_{i} M=0$ for every vertex $i$ not in $C$.

Proof. By [8, Thm. 7] $f$ induces a homeomorphism onto a closed subset. The second part is clear.

Note that for the algebra $R_{3}$ the string module $M\left(\alpha \beta^{-1} \alpha \beta \alpha^{-1}\right)$ is not in the image of the corresponding representation embedding from the Kronecker algebra $A_{2}$ to $A$-modules living on $C$.

All indecomposable pure injective modules over the algebra $B$ (of type $\widetilde{A}$ ) are described in [7], as is the topology on $\mathrm{Zg}_{B}$. Let us assume that $\mathbb{k}$ is algebraically closed. Then for every $0 \neq \lambda \in \mathbb{k}$ there is a Prüfer module $P_{\lambda}$ and an adic module $A_{\lambda}$. Besides there is a unique generic $B$-module $Q$.

To make this list complete we should include finite dimensional $B$-modules and the direct sum or direct product modules of the form $M(v)$ for (periodic) onesided strings $v$. A similar description applies to the indecomposable pure injective modules from $\mathcal{A}$.

Let $M=M(C D)$ be a string module and let $z$ be an element of the canonical basis of $M$ between $C$ and $D$. As in [10] we may introduce the pp-formula $(C$.) describing the structure of $M$ to the left from $z$, and the pp-formula (.D) describing the structure of $M$ to the right from $z$. Then $(C . D)$ will denote the conjunction of these pp-formulae. From [10, Rem. 4.1] it follows that $(M, z)$ is a free realization of $(C . D)$.

The definition of the right order $<$ on the set of finite strings may be found in [2]. For instance if $D, D^{\prime}$ are finite strings comparable within this order then $D<D^{\prime}$ iff $\left(. D^{\prime}\right)<(. D)$ as pp-formulae. Inverting this order we obtain the left order $<$.

Note that this order evidently can be extended to an order on the set of one-sided strings. Then every one-sided string $v=v_{1} \ldots$ defines a cut on the set of finite strings with first letter $v_{1}$.

In the following we will use freely the description of morphisms between indecomposable finite dimensional string modules from [3]. Precisely, every such morphism is a linear combination of graph maps, i.e. maps obtained by forming standard factors and then by standard embeddings of strings.

For instance
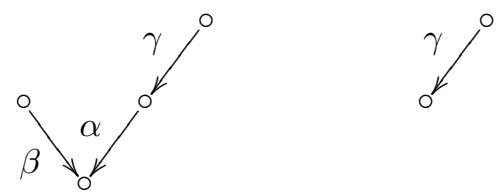

is a full list of non-simple proper factors of the string $\alpha \beta^{-1} \alpha \gamma$ over $R_{1}$.

We say that an arrow $\gamma$ is non- $C$, if $\gamma$ occurs in $C$ as neither a direct arrow nor as an inverse arrow. 
Lemma 6.2. Let $v=C^{-1} B D C$ be a string over a 1-domestic string algebra $A$ with band $C=\alpha \ldots \beta^{-1}$, where $B \neq C^{-1} \ldots, D \neq \ldots C$, and $B D$ contains a non- $C$ arrow. Let $\varphi$ be the pp-formula $\left(C^{-1} B . D C\right)$, and let the $p p$-formulae $\varphi_{i}, i=1, \ldots k$ be obtained from $\varphi$ by forming all proper factors of $v$. Then every pp-formula in the interval $\left(\varphi / \sum_{i} \varphi_{i}\right)$ is equivalent to a sum of formulae of the form $\left(E C^{-1} B . D C F\right)$ for finite strings $E, F$. In particular this interval is freely generated by two chains.

Proof. Using [10, Rem. 4.1] and the description of morphisms between string modules it is easy to prove that this interval is non-trivial.

Every pp-formula in this interval can be obtained in the following way: take a pp-formula $\psi$ below $\varphi$ and add $\sum_{i} \varphi_{i}$. Now to $\psi$ corresponds a morphism $f$ from the string module $M=M(v)$ to a finite dimensional module $N$ in which $\psi$ is realized. Let $z \in M$ be an element of the canonical basis of $M$ just to the left of $D$.

Since sums of pp-formulae are covered by our description, we may assume that $N$ is indecomposable. If $N$ is a band module then, since $B D$ contains a non- $C$ arrow, $v$ is factored properly via $f$. Then $v$ is trivialized after summing with $\sum_{i} \varphi_{i}$.

Thus we may assume that $N$ is an indecomposable string module, therefore $f$ is a linear combination of graph maps $f=\sum_{i} \lambda_{i} f_{i}$. If $v$ is factored properly via $f_{i}$, then $f_{i}$ can be deleted from this sum (without changing the resulting pp-formula after summing with $\sum_{i} \varphi_{i}$ ). Thus we may assume that each $f_{i}$ does not factor $v$ properly.

If there is only one $f_{i}\left(\right.$ with $\left.\lambda_{i} \neq 0\right)$ then $N=M\left(E C^{-1} B D C F\right), z_{i}=f(z)$ is just to the left of $D$, therefore our formula is equivalent to the formula $\left(E C^{-1} B . D C F\right)$.

Otherwise there are two different standard embeddings (maybe in different directions) of $v$ into the string $w$ defining $N$. By symmetry we may assume that $B$ contains a non- $C$ arrow.

First let us consider the possibility when both copies of $v$ are embedded in the same direction, say from left to right. So we have the following configuration in $w$ :

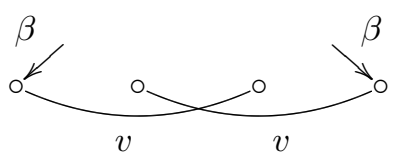

(where the intersection may be empty). By Fact 3.2 the part between $C^{-1}$ and $C^{-1}$ in the beginning of two copies of $v$ is filled by $C^{-1}$. Since $B \neq C^{-1} \ldots, B$ is contained in $C^{-1}$, a contradiction (since $B$ has a non- $C$ arrow).

Thus we may assume that two copies of $v$ are embedded in $w$ in different directions, say as $v$ and $v^{-1}$ (from left to the right). The configuration $v^{-1} \ldots v$ in $w$ leads to $C^{-1} \ldots C \ldots C^{-1}$ which contradicts Corollary 3.10. Similarly the case $v \ldots v^{-1}$ is also impossible.

So $v$ and $v^{-1}$ overlaps each other and by symmetry it suffices to consider the following configuration: 


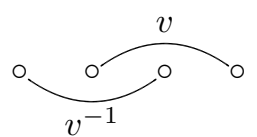

But then we clearly obtain a configuration $\gamma \gamma^{-1}$ or $\gamma^{-1} \gamma$ in the common part of $v^{-1}$ and $v$, a contradiction.

Thus every formula between $\varphi$ and $\sum_{i} \varphi_{i}$ is equivalent to a sum of formulae $\left(E C^{-1} B . D C F\right)$. Then $L_{1}=\left\{\left(E C^{-1} B.\right) \mid E C^{-1} B\right.$ is a string $\}$ is clearly a chain as is $L_{2}=\{(. D C F) \mid D C F$ is a string $\}$, and these chains generate the interval $\left(\varphi / \sum \varphi_{i}\right)$

Since arbitrary concatenation of strings in $L_{1}$ and $L_{2}$ is possible, these chains generate the corresponding interval freely.

The following theorem verifies Ringel's conjecture for 1-domestic string algebras of type $\widetilde{A}$.

Theorem 6.3. Let $M$ be an infinite dimensional indecomposable pure injective module over a 1-domestic string algebra $A$ of type $\widetilde{A}$. Then either $M$ is an (infinite dimensional) band module, or $M$ is isomorphic to a module $M(v)$, where $v$ is either a one-sided string or a two-sided (non-periodic) string.

Proof. If $e_{i} M=0$ for every vertex $i \notin C$, then $M$ is an indecomposable pure injective module over a tame hereditary finite dimensional algebra $B$ of type $\widetilde{A}$, therefore the result follows from [7, Cor. 2.10].

Thus since $M$ is indecomposable we may assume that there exists a non- $C$ arrow $\gamma$ such that $\gamma M \neq 0$. Take $0 \neq m \in \gamma M$ and let $I$ be the set of finite strings $\gamma E$ such that $m \in \gamma E M$. This set is linearly ordered with respect to $<$, so we can define a one-sided (infinite) string $\gamma v$ to be the supremum of strings in $I$. Since $M$ is pure injective $m \in \gamma v M$ (with the obvious meaning, i.e. $m \in \gamma v^{\prime} M$ for every finite substring $v^{\prime}$ of $v$ ).

We may assume that $v$ is infinite. Indeed otherwise $M$ is a one-directed pure injective module in the terminology of [10], therefore the description of $M$ follows from [10, Thm. 5.4].

Similarly we may assume that either $m \in \delta M$ for an arrow $\delta \neq \gamma$, or $\delta m \neq 0$ for some arrow $\delta$ such that $\delta \gamma \neq 0$. Let $J$ be the set of strings $D \delta^{-1}(D \delta)$ such that $m$ is divided by this string 'on the left'. As above if a one-sided string $w$ is the supremum of $J$, then $m$ is divided by $w$ on the left, and we may assume that $w$ is infinite. Thus $w v$ is a two-sided string.

By Lemma 3.8 we may write $w v$ in the form ${ }^{\infty} C^{-1} B D C^{\infty}$, where $D$ begins with $\gamma$ and $B$ ends with $\delta$ or $\delta^{-1}$.

Suppose that we have performed this construction for every nonzero $m \in \gamma M$. Compare those strings ${ }^{\infty} E^{-1} B D E^{\infty}$ appearing ( $D$ begins with $\gamma$ and $E=C$ or $\left.E=C^{-1}\right)$ with respect to the product order $\left(<^{\prime},<\right)$. Since by Corollary 3.9 there are only finitely many two-sided strings over $A$, we may assume that the string defined by $m$ is maximal with respect to this order. 
Then clearly $M$ is in the interval $\left(\varphi / \sum_{i} \varphi_{i}\right)$, where $\varphi$ and $\varphi_{i}$ are as in Lemma 6.2, which is freely generated by the chains $L_{1}$ and $L_{2}$. By Lemma 4.9 , the cut on this interval defined by the pp-type $p=p p_{M}(m)$ is uniquely determined by cuts on $L_{1}$ and $L_{2}$, i.e. by the strings $w$ and $v$. Let $N=M(w v)$ be the (direct sum, direct product or mixed) module from Ringel's list corresponding to $w v$. Let $n$ be between $w$ and $v$ in $N$, and let $q=p p_{N}(n)$.

Since (see [10, L. 4.2]) $q$ is homogeneous, it is easy to check that $q \in\left(\varphi / \sum \varphi_{i}\right)$. Also $q$ defines the same (as $p$ ) cut on $L_{1}$ and $L_{2}$. Then $M \cong N$ by Proposition 4.10.

\section{KRULL-GABRIEL Dimension}

For the precise definition of Krull-Gabriel dimension of a finite dimensional algebra $A, \operatorname{KG}(A)$, see [18].

We will use the following equivalent to this notion.

Fact 7.1. [9, Prop. 2.3] The Krull-Gabriel dimension of a finite dimensional algebra $A$ is equal to the $m$-dimension of the lattice of all pp-formulae over $A$.

Unfortunately for a string algebra $A$, the lattice of all pp-formulae over $A$ has a very complex structure, so we believe that it may be very difficult to calculate its $m$-dimension by 'bare hands'. Therefore the following trick is very useful. Suppose that we have described (in some terms) indecomposable pure injective modules over $A$. After that it is usually not difficult to check the so-called isolation property over $A$ : every isolated point in any theory of $A$-modules is isolated by a minimal pair. Then [6, Prop. 10.19] implies that the $m$-dimension of the lattice of all pp-formulae over $A$ is the same as the Cantor-Bendixson rank of $\mathrm{Zg}_{A}, \mathrm{CB}\left(\mathrm{Zg}_{A}\right)$. And the last invariant can be calculated locally.

We are going to follow this route.

Fact 7.2. [10, Cor. 7.4] Let $A$ be a domestic string algebra and let $n$ be the maximal length of a path in the bridge quiver of $A$. Then $\mathrm{CB}\left(\mathrm{Zg}_{A}\right) \geq n+2$.

Lemma 7.3. Let $A$ be a 1-domestic string algebra of type $\widetilde{A}$ with band $C=\alpha \ldots \beta^{-1}$ whose bridge quiver is the disjoint union of $C$ and $C^{-1}$ (that is, every two-sided string over $A$ is periodic). Then $\mathrm{CB}\left(\mathrm{Zg}_{A}\right)=2$.

Proof. The lower bound follows from Fact 7.2.

Let $M$ be an infinite dimensional indecomposable pure injective $A$-module such that $\gamma M \neq 0$ for some non- $C$ arrow $\gamma$. By Theorem $6.3 M$ is isomorphic to a module $M(v)$, where $v$ is a one-sided string, in particular $M$ is one-directed in the terminology of [10].

Then by $[10$, Thm. 7.3] we obtain that $\mathrm{CB}(M) \leq 1$, therefore $\mathrm{CB}(M)=1$ since $M$ is infinite dimensional. Thus all these points are removed from the Ziegler spectrum at level 1 . 
Let $N=M(C, \lambda, 1)$ be a finite dimensional band module corresponding to $C$. Let $m$ be the element of the canonical basis of $N$ between $\beta^{-1}$ and $\alpha$, and let $\varphi$ generate the pp-type of $m$ in $N$. We prove that the pair $(\varphi / x=0)$ isolates the Prüfer module $P_{\lambda}$ in $\mathrm{Zg}_{A}$ at level 1. Since $N$ is a submodule of $P_{\lambda}$, this pair opens on $P_{\lambda}$.

Let $B$ be the tame hereditary finite dimensional algebra corresponding to $A$. From the corresponding result for $B$ we know that this pair separates $P_{\lambda}$ from all other Prüfer points and all adic points in $\mathcal{A}$.

So it suffices to show that this separates $P_{\lambda}$ from all points $M=M(v)$, where $v$ is a one-sided (non-periodic) string. Otherwise there is a morphism $f$ from $N$ to $M$ such that $f(m) \neq 0$. By symmetry we may assume that $v=D C^{\infty}$ where $D$ does not contain $C$ as a substring. By Lemma 3.6 and Fact $3.2 D$ does not contain $\alpha$ as a direct arrow.

Clearly $M(D)$ is a submodule of $M$. Then, combining $f$ with the projection $M \rightarrow M / M(D)$, we obtain a homomorphism $\bar{f}$ from $N$ to the (degenerated) adic or Prüfer module $M(w)$. From the corresponding result for $B$-modules, $\bar{f}$ will be zero.

Thus the image of $f$ is contained in $M(D)$. Since $f(m) \neq 0$, therefore $D$ contains either a substring $\beta^{-1} \alpha$ or a substring $\alpha^{-1} \beta$, and the first case is impossible by the above remark. In the second case we obtain a two-sided string ${ }^{\infty} C^{-1} \beta \ldots C^{\infty}$ over $A$, which contradicts the assumption.

Thus every Prüfer point $P_{\lambda}$ is isolated at level 1. By elementary duality (see [9]) the same is true for every adic point $A_{\lambda}$.

After removing all these points at level 1, by Theorem 6.3 at level 2 there remains only the generic point $Q$. Thus $\mathrm{CB}\left(\mathrm{Zg}_{A}\right)=2$.

Proposition 7.4. Let $A$ be a 1-domestic string algebra of type $\widetilde{A}$ with band $C=$ $\alpha \ldots \beta^{-1}$ such that the bridge quiver of $A$ contains an arrow $C^{-1} \rightarrow C$. Then $\mathrm{CB}\left(\mathrm{Zg}_{A}\right)=3$.

Proof. By Fact 7.2 we obtain $\mathrm{CB}\left(\mathrm{Zg}_{A}\right) \geq 3$.

By similar arguments (see [10]) every one-directed point in $\mathrm{Zg}_{A}$ has CB-rank not more then 2 , for instance $M\left(C^{-\infty}\right)$ has CB-rank 2 by calculations in [10, Thm. 7.3]. Moreover all non-periodic one-directed points have CB-rank 1.

Let $M=M\left({ }^{\infty} C^{-1} D C^{\infty}\right)$, where $D$ contains a non- $C$ arrow $\gamma$ and $D$ is minimal with this property. Let $m$ be an element in the canonical basis of $M$ such that $m \in \gamma M$ and $p=p p_{M}(m)$.

Choose a distributive interval opening $M$ as in the proof of Theorem 6.3. By Lemma 5.2 the CB-rank of $M$ is equal to the sum of the $m$-dimensions of the cuts $p_{1}$ and $p_{2}$ defined by $p$ on chains generating this interval. But it is easy to calculate (see $[10$, Thm. 7.3]) that the $m$-dimension of both cuts is 1 , therefore $\operatorname{CB}(M)=2$. 
We prove that every Prüfer point $P_{\lambda}, 0 \neq \lambda \in \mathbb{k}$, has CB-rank not more than 2. From the above remarks it follows that it suffices to separate $P_{\lambda}$ from one-sided periodic points and from two-sided points $M(v)$.

Choose the band module $N$ and the pair $(\varphi / x=0)$ as in the proof of Lemma 7.3. As there this pair separates $P_{\lambda}$ from every one-sided periodic point. So it suffices to check that this pair separates $P_{\lambda}$ from any two-sided point $M=M(v)$, where $v={ }^{\infty} C^{-1} D C^{\infty}, D \neq C^{-1} \ldots, D \neq \ldots C$ and $D$ contains a non- $C$ arrow $\gamma$.

Indeed otherwise there exists a morphism $f$ from $N$ to $M$ such that $f(m) \neq 0$. Clearly $M(D)$ is a submodule of $M$, and the factor $M / M(D)$ is the direct sum of two modules $M\left(v_{i}\right)$ with almost periodic strings $v_{i}$. Then as the proof of Lemma 7.3, the image of $f$ is contained in $M(D)$.

Thus either $\beta^{-1} \alpha$ or $\alpha^{-1} \beta$ is a substring of $D$. In the first case we have a configuration $\alpha \ldots C^{\infty}$ in $v$, therefore $D=D^{\prime} C$, a contradiction. Similarly if $\alpha^{-1} \beta$ is a substring of $D$ we obtain a contradiction by considering $C^{-1}$ in the left hand part of $v$.

Thus the CB-rank of every Prüfer point $P_{\lambda}$ is $\leq 2$. By elementary duality the same is true for every $\lambda$-adic point.

After removing all these points on level 2 we are left with just the one, generic, point $Q$, which therefore has CB-rank $\leq 3$.

Before calculating the Krull-Gabriel dimension let us recall the following fact.

Fact 7.5. [9, Thm. 2.4] If $\operatorname{KG}(A)<\infty$ (i.e. if the lattice of all pp-formulae over $A$ has m-dimension $)$, then $\mathrm{CB}\left(\mathrm{Zg}_{A}\right)=\mathrm{KG}(A)$.

Let $T$ be any theory of $A$-modules and let $\mathrm{Zg}_{T}$ be the closed subset of the Ziegler spectrum corresponding to $T$. Then, similarly to the Cantor-Bendixson analysis, we may organize the 'minimal' analysis of $\mathrm{Zg}_{T}$ by iterated removal of points isolated by minimal pairs. We obtain a corresponding minimal rank, $\operatorname{minrk}(T)$, on points of $\mathrm{Zg}_{T}$.

Lemma 7.6. If $\operatorname{minrk}(T)<\infty$, then $T$ has $m$-dimension. Therefore $\mathrm{CB}\left(\mathrm{Zg}_{T}\right)=$ $\mathrm{KG}(T)$.

Proof. Similar to [6, Prop. 10.19] at every step of the minimal analysis we factorize the lattice of pp-formulae of $T$ by a the congruence generated by (certain) intervals of finite length.

Thus this analysis runs no faster than the $m$-dimension analysis on the lattice of pp-formulae of $T$. It follows that $\operatorname{mdim}(T) \leq \operatorname{minrk}(T)$, therefore $\operatorname{KG}(T)=$ $\operatorname{mdim}(T)=\mathrm{CB}\left(\mathrm{Zg}_{T}\right)$ by Fact 7.5 .

Theorem 7.7. Let $A$ be a 1-domestic string algebra of type $\widetilde{A}$ with band $C$.

1) If the bridge quiver of $A$ does not contain an arrow $C^{-1} \rightarrow C$ then $\operatorname{KG}(A)=2$.

2) If the bridge quiver of $A$ contains an arrow $C^{-1} \rightarrow C$ (that is, if there is a two-sided nonperiodic string over $A$ ) then $\mathrm{KG}(A)=3$. 
Proof. Let $T$ be the theory of all $A$-modules. In both cases we prove that $\operatorname{minrk}(T)$ is finite. Then the result will follow by Lemma 7.6, Lemma 7.3 and Proposition 7.4.

Note that every one-directed or two-directed module $M(v)$ opens on a distributive interval. Therefore (see proof of Lemma 5.2) if it isolated on some level, it is isolated by a minimal pair.

At the zero level all finite dimensional points are isolated by a minimal pair, so we can remove them. At level 1 we may remove at least all one-directed points of CB-rank 1.

Similarly at level 2 we may remove all points of the form $M(v)$. The remaining part is a closed subset of $\mathcal{A} \cong \operatorname{Zg}(B)$, therefore a minimal analysis can be finished in at most two steps.

Corollary 7.8. Let $A$ be a 1-domestic string algebra of type $\widetilde{A}$. Then there exists no superdecomposable pure injective module over $A$.

Proof. By Theorem 7.7 the $m$-dimension of the lattice of all pp-formulae over $A$ is finite.

\section{REFERENCES}

[1] K. Burke, M. Prest, The Ziegler and Zariski spectra of some domestic string algebras, Algebr. Represent. Theory, 5 (2002), 211-234.

[2] M. Butler, C.M. Ringel, Auslander-Reiten sequences with few middle terms and applications to string algebras, Comm. Algebra, 15(1-2) (1987), 145-179.

[3] W.W. Crawley-Boevey, Maps between representations of zero relation algebras, J. Algebra, 126 (1989), 259-263.

[4] W. Geigle, The Krull-Gabriel dimension of the representation theory of a tame hereditary artin algebra and applications to the structure of exact sequences, Manuscripta Math., 54 (1985), 83-106.

[5] G. Grätzer, General Lattice Theory. Academie Verlag, 1978.

[6] M. Prest, Model Theory and Modules. London Math. Soc. Lecture Note Series, Cambridge University Press, 130 (1987).

[7] M. Prest, Ziegler spectra of tame hereditary finite dimensional algebras, J. Algebra, 207 (1998), 146-164.

[8] M. Prest, Representation embeddings and the Ziegler spectrum, J. Pure Appl. Math., 113 (1996), 315-323.

[9] M. Prest, Topological and geometric aspects of the Ziegler spectrum, pp. 369-392 in: Trends in Math., Birkhäuser, 2000.

[10] M. Prest, G. Puninski, One-dircted indecomposable pure injective modules over string algebras, Colloq. Math., 101 (2004), 89-112.

[11] M. Prest, J. Schröer, Serial functors, Jacobson radical and representation type, J. Pure Appl. Algebra, 170 (2002), 295-307.

[12] G. Puninski, Superdecomposable pure-injective modules exist over some string algebras, Proc. Amer. Math. Soc., 132 (2004), 1891-1898.

[13] G. Puninski, The Krull-Gabriel dimension of a serial ring, Comm. Algebra, 31(12) (2003), $5977-5993$.

[14] G. Reynders, Ziegler spectrum of a serial ring with Krull dimension, Comm. Algebra, 27(6) (1999), 2583-2611. 
[15] C.M. Ringel, Some algebraically compact modules. I, pp. 419-439 in: Abelian Groups and Modules, eds. A. Facchini, C. Menini, Kluwer, 1995.

[16] C.M. Ringel, Infinite length modules. Some examples as introduction, pp. 1-73 in: Infinite Length Modules, H. Krause, C.M. Rindel eds., Trends in Math., Birkhäuser, 2000.

[17] J. Schröer, Hammocks for string algebras, Sonderforshungsbereich 343, Ergänzungsreihe $97-$ 010, Universität Bielefeld, 1997.

[18] J. Schröer, On the Krull-Gabriel dimension of an algebra, Math. Z., 233 (2000), 287-303.

[19] J. Schröer, On the infinite radical of a module category, Proc. London Math. Soc. (3), 81 (2000), 651-674.

[20] A.A. Tuganbaev, Semidistributive Rings and Modules. Kluwer, Mathematics and its applications, 1998.

[21] M. Ziegler, Model theory of modules, Ann. Pure Appl. Logic, 26 (1984), 149-213.

School of Mathematics, University of Manchester, Lamb Bullding, Booth Street East, Manchester, M13 9PL, Great Britain

E-mail address: mprest@maths.man.ac.uk

E-mail address: gpuninski@maths.man.ac.uk 